\title{
Prediction Partial Molar Heat Capacity at Infinite Dilution for Aqueous Solutions of Various Polar Aromatic Compounds over a Wide Range of Conditions Using Artificial Neural Networks
}

\author{
Aziz Habibi-Yangjeh ${ }^{\star}$ and Mahdi Esmailian \\ Department of Chemistry, Facultw of Science, Enwersity of Mohaghegh Ardabili. P.O. Box 179. Ardabil. Iran \\ E-mail: ahabibiàma acir: habibivangjehovahoo.com \\ Received December 21, 2006
}

\begin{abstract}
Artificial neural networks (ANNs). for a first time. were successfully developed for the prediction partial molar heat capacity of aqueous solutions at infinite dilution for various polar aromatic compounds over wide range of temperatures (303.55-623.20 K) and pressures (0.1-30.2 MPa). Two three-layered feed forward ANNs with back-propagation of error were generated using three (the heat capacity in $\mathrm{T}=303.55 \mathrm{~K}$ and $\mathrm{P}=0.1 \mathrm{MPa}$, temperature and pressure) and six parameters (four theoretical descriptors. temperature and pressure) as inputs and its output is partial molar heat capacity at infinite dilution. It was found that properly selected and trained neural networks could fairly represent dependence of the heat capacity on the molecular descriptors, temperature and pressure. Mean percentage deviations (MPD) for prediction set by the models are 4.755 and 4.642, respectively
\end{abstract}

Key Words : Artificial neural networks. Partial molar heat capacity. Aqueous solutions. Polar aromatic compounds. Theoretical descriptors

\section{Introduction}

Heat capacities of organic solutes in water are of great interest for calculating thermodynamic properties of organic aqueous systems at super ambient conditions. The temperature integration of the heat capacity data allows obtaining the standard chenical potentials and activity coefficients needed for calculating phase and chenical equilibria at conditions of interest for geochenistry. power cycle chemistry and hydrothernal techuologies. ${ }^{\text {I. }}$ Only a limited amount of data are available at upper temperatures and pressures. The main reason is certainly a time consuning and costly task of the calorimeter construction since commercial instruments allowing the heat capacity determination over a range of temperatures do not have the precision necessary for the calculation of heat capacity. For this reason, it is very valuable to predict the heat capacity at higher temperatures and pressures using mininum number of experiments. The prediction of physicochenical and biological properties/activities for organic molecules is the main objectives of the quantitative structure-property/ activity relationships (QSPRs/QSARs).-9 ${ }^{3-9}$ QSPRQSAR models are obtained on the basis of the correlation between the experimental values of the property/activity and descriptors reflecting the molecular structure of the respective conpounds. Since these theoretical descriptors are determined solely from computational methods. a priori predictions of the properties/activities of compounds are possible. no laboratory measurements are needed thus saving time. space. materials, equipment and alleviating safety (toxicity) and disposal concerns. ${ }^{11.11}$

Various methods for constructing QSPR/QSAR models have been used including multi-parameter linear regression (MLR), principal component analysis (PCA) and partial least-squares regression (PLS). ${ }^{12-15}$ In addition. artificial neural networks (ANNs) have become popular due to their success where complex non-linear relationships exist amongst data. ${ }^{16-18}$ ANNs are biologically inspired computer programs designed to simulate the way in which the human brain processes information. ${ }^{18}$ ANNs gather their knowledge by detecting the patterns and relationships in data, not from programming. The wide applicability of ANNs stems from their flexibility and ability to model non-linear systems without prior knowledge of an empirical model. For these reason in recent years. ANNs have been used to a wide variety of chemical problems such as simulation of mass spectra. ion interaction chromatography, aqueous solubility and partition coefficient. simulation of nuclear magnetic resonance spectra, prediction of bioconcentration factor. solvent effects on reaction rate. prediction nomalized polarity parameter in mixed solvent systems, acidity constant of organic compounds and dielectric constant of binary mixtures. $^{\text {19-4] }}$

In this work an ANN model. for a first time. was generated for prediction partial molar heat capacity of aqueous solutions at infinite dilution for various polar aromatic compounds over wide range of temperatures (303.55-623.20 $\mathrm{K})$ and pressures (0.1-30.2 $\mathrm{MPa}$ ) using three inputs (the partial molar heat capacity at infinite dilution for the various aqueous solutions at $\mathrm{T}=303.55 \mathrm{~K}$ and $\mathrm{P}=0.1 \mathrm{MPa}$, temperature and pressure). In the next step, a MLR model was constructed between the heat capacity of the compounds and four theoretical descriptors. Then an ANN model using the theoretical descriptors. temperature and pressure was con- 
structed for prediction the heat capacity and the results were compared with the experimental values of them.

\section{Methods and Procedure}

Data set. A reliable database is critically important for the training of ANNs. Very recently partial molar heat capacity at infinite dilution have been determined for different aqueous solutions of polar aromatic compounds at various temperatures and pressures. ${ }^{1,2}$ In this work, the data for aqueous solutions of phenol. $o$-cresol. $m$-cresol. $p$-cresol. aniline, $o$-toluidne, $m$-toluidine, $p$-toluidine. m-aninophenol and $o$-diaminobenzene that they have at least eight values for the heat capacity at various temperatures and pressures have been used as data set. The data set was randomly divided into three groups: a training set. a validation set and a prediction set consisting of 74.21 and 21 data, respectively. ${ }^{18.19}$ The training and validation sets were used for the model generation and the prediction set was used for evaluation of the generated model, because a prediction set is a better estimator of the ANN generalization ability than a monitoring (validation) set.

Descriptor generation. In order to calculate the theoretical descriptors. the z-matrices (molecular models) were constructed with the aid of HyperChem 7.0 and molecular structures were optimized using AMI algorithm. ${ }^{4 \hat{2}}$ In order to calculate theoretical descriptors. the molecular geometries of molecules were further optimized by Dragon package version $2.1{ }^{+3}$ For this purpose the output of the HyperChem software for each compound fed into the Dragon program and the descriptors were calculated. As a result. a total of 1481 theoretical descriptors were calculated for each compound in the data sets ( 11 compounds).

Feature selection. The theoretical descriptors were reduced by the following methods: 1) descriptors that are constant or nearly constant have been eliminated. because these descriptors can not define the variation of the property with structure: 2) in order to decrease the redundancy existing in the descriptors data matrix the correlation coefficients for all pairs of remaining descriptors were determined. If a correlation coefficient was higher than 0.91 . the descriptor with lower correlation with the heat capacity was eliminated: ${ }^{+4,+5^{5}} 3$ ) the method of stepwise multi-parameter linear regression was used to select the most important descriptors and to calculate the coefficients relating the heat capacity to the descriptors. ${ }^{15}$ The MLR models were generated using $\mathrm{spss} / \mathrm{pc}$ software package release $10.0 .{ }^{\text {t6 }}$

Neural network generation. The specification of a typical neural network model requires the choice of the type of inputs. the number of hidden layers. the number of neurons in each hidden layer and the connection structure between the inputs and the output layers. Three-layer networks with sigmoidal transfer function for neurons were designed. The initial weights were randomly selected between 0 and 1 . Before training. the input and output values were normalized between 0.1 and 0.9 . The optimization of the weights and biases was carried out according to the resilient back-propagation algoritlun. ${ }^{46}$ For evaluation predictive power of the networks. the trained ANNs were used to predict the heat capacity for 21 aqueous solutions included in the prediction set. The perfornances of ANNs are evaluated by the mean percentage deviation (MPD) and root-mean square error (RMSE). which are defined as follows:

$$
\begin{aligned}
& \mathrm{MPD}=\frac{100}{N} \sum_{i=1}^{N}\left|\frac{\left(P_{i}^{\text {calc }}-P_{i}^{\text {exp }}\right)}{P_{i}^{\text {exp }}}\right| \\
& \mathrm{RMSE}=\sqrt{\sum_{i=1}^{N} \frac{\left(P_{i}^{\text {calc }}-P_{i}^{\text {exp }}\right)^{2}}{N}}
\end{aligned}
$$

where $P_{i}^{\text {exp }}$ and $P_{i}^{c a l}$ are experimental and calculated values of the heat capacity using the models.

Individual percent deviation (IPD) is defined as follows:

$$
\mathrm{IPD}=100 \times\left(\frac{P_{i}^{\text {calc }}-P_{i}^{\text {exp }}}{P_{i}^{\exp }}\right)
$$

The processing of the data was carried using Matlab $6.5 .^{47}$ The neural networks were implemented using Neural Network Toolbox Ver. 4.0 for Matlab. ${ }^{1 \$}$

\section{Results and Discussion}

Prediction the heat capacity without theoretical descriptors. There are no theoretical principles for choosing the proper network topology: so different structures were tested in order to obtain the optimal hidden neurons and training cycles. ${ }^{18.19}$ Before training the network. the numbers of nodes in the hidden layer were optimized. In order to optimize the number of nodes in the hidden layer. several training sessions were conducted with different numbers of hidden nodes (from one to eleven). The root mean squared error of training (RMSET) and validation (RMSEV) sets were plotted versts the number of iterations for different number of neurons at the hidden layer and the minimum value of RMSEV was recorded as the optimum value. Plot of RMSET and RMSEV versus the number of nodes in the hidden layer has been demonstrated in Figure 1. It is clear that nine nodes in hidden layer is optimum value.

This network consists of three inputs including the partial molar heat capacity at infinite dilution for the various aqueous solutions (at $\mathrm{T}=303.55 \mathrm{~K}$ and $\mathrm{P}=0.1 \mathrm{MPa}$ ). temperature and pressure. Then an ANN with architecture 39-1 was generated. It is note wortlyy that training of the network was stopped when the RMSEV started to increases i.e. when overtraining begins. The overtraining causes the ANN to loose its prediction power. ${ }^{32}$ Therefore. during training of the networks. it is desirable that iterations are stopped when overtraining begins. To control the overtraining of the network during the training procedure, the values of RMSET and RMSEV were calculated and recorded to monitor the extent of the learning in various iterations. 


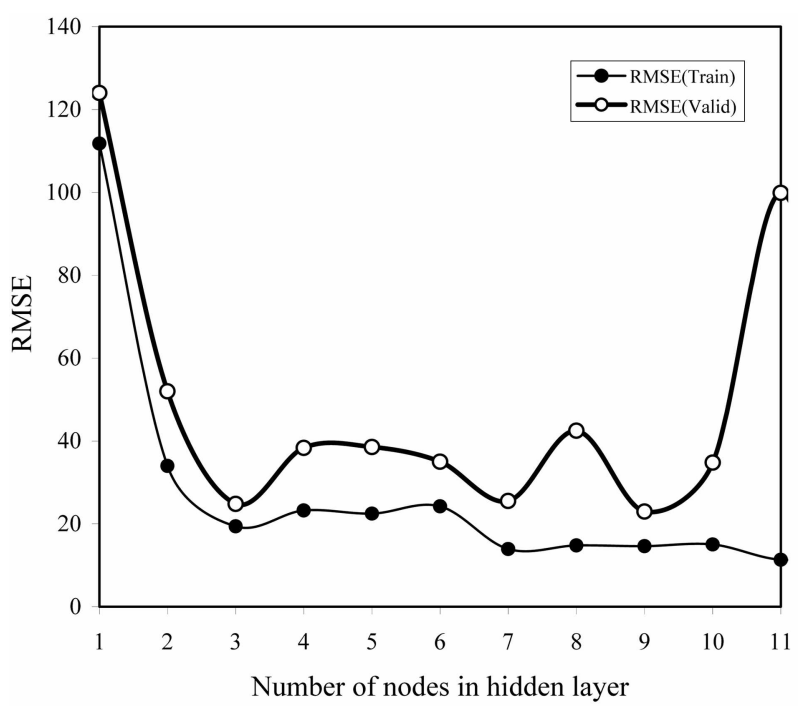

Figure 1. Plot of RMSE, for training and validation sets versus the number of nodes in hidden layer.

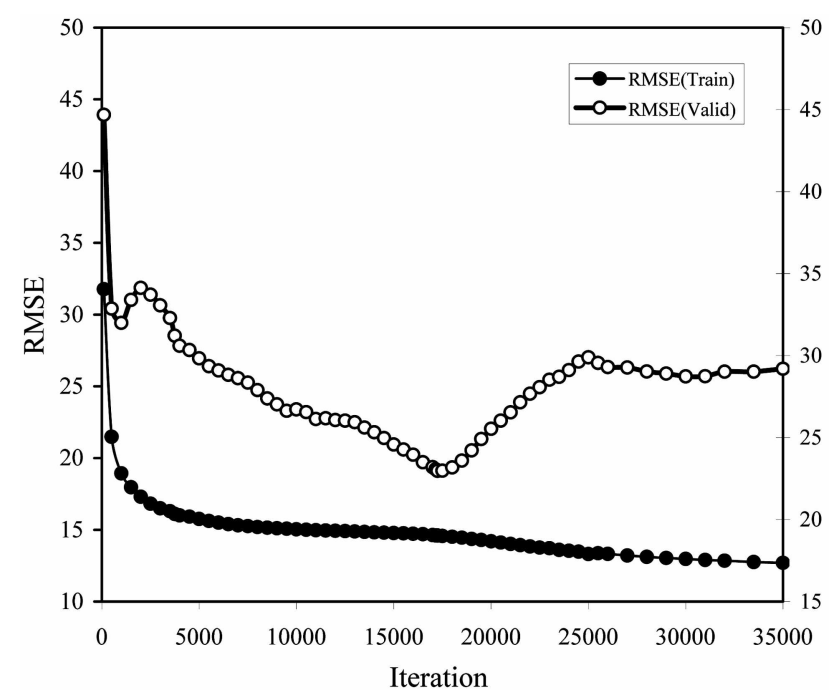

Figure 2. Plot of RMSE for training and validation sets (for the AN. model with architecture 3-9-1) versus the number of iterations.

Results obtained showed that after 17250 iterations the value of RMSEV started to increase and overfitting began (Figure 2).

The generated ANN was then trained using the training set for optimization of the weights and biases. For evaluation predictive power of the generated ANN, an optimized network was applied for prediction the heat capacity of different aqueous solutions at various temperatures and pressures in the prediction set, which were not used in the modeling procedure. Values of partial molar heat capacity for different aqueous solutions of various polar aromatic compounds along with the calculated and IPD values at various temperatures and pressures for training, validation and prediction sets have been shown in 'Jable 1 .

The correlation equation for all of the calculated values of the heat capacity from the ANN model and the experimental values is as follows:

$$
\begin{gathered}
C_{p, 2}^{\prime \prime}(\mathrm{cal})=0.9606 \mathrm{C}_{p, 2}^{\prime \prime}(\mathrm{exp})-13.849 \\
\mathrm{~N}-116 ; \mathrm{R}-0.9859 ; \mathrm{MPD}-3.017 \\
\mathrm{RMSE}-19.642 ; \mathrm{F}-3950.35
\end{gathered}
$$

Similarly, the correlation of $C_{p, 2}^{\prime \prime}$ (cal) values versus $C_{p .2}^{\prime 2}$ (exp) in prediction set gives equation (5):

$$
\begin{gathered}
\mathrm{C}_{p, 2}^{\prime \prime}(\mathrm{cal})-0.9899 \mathrm{C}_{p, 2}^{\prime \prime}(\exp )-11.531 \\
\mathrm{~N}=2 \mathrm{l} ; \mathrm{R}=0.9761 ; \mathrm{MPD}=4.755 \\
\mathrm{RMS} F=29.179 ; \mathrm{F}=383.29
\end{gathered}
$$

As can be seen the calculated values of the heat capacity are in good agreement with those of the experimental values. Plot of IPD for $C_{p, 2}^{\prime \prime}$ values in prediction set versis the experimental values of it has been illustrated in Figure 3. The results demonstrate that the MPD value for $C_{p .2}^{\prime \prime}$ values in the prediction set is 4.755 . As can be seen the model did not show proportional and systematic error, because the propagation of errors in both sides of zero are random (Figure 3).

The correlation coefficient (R), RMSE. MPD and statistical F-value of the model for total, training, validation and prediction sets show potential of the ANN model for simulation the complicated nonlinear relationship between the partial molar heat capacity at infinite dilution for aqueous solutions of the various polar aromatic compounds on the heat capacity in $\mathrm{T}-303.55 \mathrm{~K}$ and $\mathrm{P}-0.1 \mathrm{MPa}$, temperature and pressure (Table 2).

Prediction the heat capacity using theoretical descriptors. After feature selection (see section methods and procedure). multi-parameter linear correlation of the heat capacity verstis the molecular descriptors in the training set gives the results in Table 3. It can be seen that four descriptors are appeared in the MLR model. These descriptors are: complementary information content (neighborhood symmetry of 0 -order) (CICO), geary autocorrelation-lag3/ weighted by atomic masses (GATS3m), radia distribution function-5.0/weighted by atomic masses (RDF050m) and 3D-MoRSF-signal 08/weighted by atomic polarizabilities (Mor08p).

The correlation equation for the calculated values of $\mathrm{C}_{t: 2}^{\prime \prime}$ versus the experimental values is as follows:

$$
\begin{gathered}
\mathrm{C}_{p .2}^{\prime \prime}(\mathrm{cal})=0.9993 \mathrm{C}_{p, 2}^{\prime 2}(\exp )-0.2194 \\
\mathrm{~N}-10 ; \mathrm{R}-0.99968 ; \mathrm{MPD}-0.1819 ; \\
\text { RMSE }-0.7938 ; \mathrm{F}-21880.83
\end{gathered}
$$

The next step in this work is the generation of the ANN model using theoretical descriptors. The artificial neural network consists of six inputs (including four descriptors appearing in the MLR model, temperature and pressure) and one output for $\mathrm{C}_{p, 2}^{\prime \prime}$. Plot of RMSE'T and RMSEV versus the number of nodes in the hidden layer has been demonstrated in Figure 4. It is clear that three nodes in hidden layer is optimum value.

Then an ANN with architecture 6-3-1 was generated. Io 
Table 1. Values of partial molar heat capacity at intinite dilution for aqueous solutions of various polar aromatic compounds along with the calculated and IPD (individual percent deviation) at various temperatures and pressures using the ANN models

\begin{tabular}{|c|c|c|c|c|c|c|c|c|c|}
\hline No. & Aqueous solutions & Data set & $\mathrm{T}$ & $\mathrm{P}$ & $C_{p .2}^{0}(\exp )$ & $\mathrm{C}_{p .2}^{o}(\mathrm{cal})^{p}$ & $\mathrm{IPD}^{a}$ & $\mathrm{C}_{p .2}^{o}(\mathrm{cal})^{b}$ & $\mathrm{IPD}^{b}$ \\
\hline 1 & phenol & training & 303.55 & 0.1 & 325.1 & 313.19 & -3.66 & 318.06 & -2.17 \\
\hline 2 & phenol & validation & 372.23 & 2.2 & 312.3 & 320.64 & 2.67 & 325.15 & 4.11 \\
\hline 3 & phenol & training & 422.61 & 2.1 & 314.8 & 323.88 & 2.88 & 334.24 & 6.18 \\
\hline 4 & phenol & training & 473.54 & 2.1 & 327.6 & 329.08 & 0.45 & 347.02 & 5.93 \\
\hline 5 & phenol & prediction & 523.52 & 5 & 364.7 & 359.64 & -1.39 & 366.56 & 0.51 \\
\hline 6 & phenol & training & 573.38 & 10 & 467.5 & 458.76 & -1.87 & 454.17 & -2.85 \\
\hline 7 & phenol & training & 574.58 & 10.2 & 463.4 & 463.35 & -0.01 & 458.31 & -1.10 \\
\hline 8 & phenol & validation & 598.22 & 13.3 & 626.8 & 581.99 & -7.15 & 615.72 & -1.77 \\
\hline 9 & phenol & training & 524.37 & 10.1 & 354.4 & 360.9 & 1.83 & 354,09 & -0.09 \\
\hline 10 & phenol & training & 573.3 & 20.2 & 382.7 & 378.46 & -1.11 & 375.34 & -1.92 \\
\hline 11 & phenol & training & 598.17 & 20.2 & 463.9 & 469.32 & 1.17 & 468.58 & 1.01 \\
\hline 12 & phenol & training & 474.89 & 30.3 & 311 & 323.5 & 4.02 & 316.75 & 1.85 \\
\hline 13 & phenol & training & 523.6 & 30.1 & 324.1 & 326.23 & 0.66 & 323.42 & -0.21 \\
\hline 14 & phenol & validation & 573.22 & 30 & 346.9 & 349.56 & 0.77 & 342.33 & -1.32 \\
\hline 15 & phenol & training & 573.95 & 30 & 350.6 & 350.17 & -0.12 & 342.89 & -2.20 \\
\hline 16 & phenol & training & 598.2 & 30.4 & 374.9 & 371.1 & -1.01 & 374.58 & -0.09 \\
\hline 17 & phenol & prediction & 623.2 & 30.2 & 446.8 & 397.94 & -10.94 & 497.17 & 11.27 \\
\hline 18 & $o$-cresol & training & 303.55 & 0.1 & 407 & 406.84 & -0.04 & 400.39 & -1.62 \\
\hline 19 & $o$-cresol & validation & 372.23 & 2.2 & 384 & 402.77 & 4.89 & 405.78 & 5.67 \\
\hline 20 & $o$-cresol & training & 422.61 & 2.1 & 389.6 & 397.15 & 1.94 & 409.38 & 5.08 \\
\hline 21 & $O$-cresol & training & 473.53 & 2.1 & 416.5 & 406.96 & -2.29 & 415.31 & -0.29 \\
\hline 22 & $o$-cresol & prediction & 523.51 & 5 & 461.9 & 441.38 & -4.44 & $\$ 4.79$ & -3.70 \\
\hline 23 & $o$-cresol & training & 573.39 & 10.1 & 623.3 & 624.79 & 0.24 & $6+2.81$ & 3.13 \\
\hline 24 & $O$-ctesol & training & 574.28 & 10.1 & 616 & 630.64 & 2.38 & 652.91 & 5.99 \\
\hline 25 & 0 -CTESOl & training & 598.22 & 13.3 & 939.3 & 889.62 & -5.29 & 898.77 & -4.32 \\
\hline 26 & $o$-cresol & training & 524.37 & 10.1 & 439.9 & 451.93 & 2.73 & 432.55 & -1.67 \\
\hline 27 & $o$-cresol & training & 573.3 & 20.2 & 513.9 & 513.44 & -0.09 & 497.34 & -3.22 \\
\hline 28 & $o$-cresol & prediction & 598.17 & 20.3 & 676.9 & 658 & -2.79 & 702.49 & 3.78 \\
\hline 29 & $o$-cresol & training & 474.89 & 30.3 & 383.6 & 386.4 & 0.73 & 398.96 & 4.00 \\
\hline 30 & $o$-cresol & training & 523.6 & 30.3 & 397.1 & 398.5 & 0.35 & 407.29 & 2.57 \\
\hline 31 & $o$-cresol & validation & 573.23 & 30.1 & 411.4 & 447.07 & 8.67 & 441.72 & 7.37 \\
\hline 32 & $o$-cresol & training & 574.04 & 30 & 436.2 & 448.91 & 2.91 & 443.46 & 1.67 \\
\hline 33 & $o$-cresol & training & 598.2 & 30.4 & 558.6 & 519.84 & -6.94 & 520.12 & -6.89 \\
\hline 34 & $o$-cresol & prediction & 623.2 & 30.1 & 747.9 & 670.06 & -10.41 & 786.70 & 5.19 \\
\hline 35 & $m$-cresol & training & 303.55 & 0.1 & 397.3 & 394.61 & -0.68 & 389.87 & -1.87 \\
\hline 36 & $m$-cresol & validation & 372.24 & 2.2 & 363.8 & 392.58 & 7.91 & 398.54 & 9.55 \\
\hline 37 & $m$-cresol & training & 422.62 & 2.1 & 385.8 & 395.54 & 2.52 & 404.36 & 4.81 \\
\hline 38 & $m$-cresol & training & 473.53 & 2.1 & 399.5 & 416.8 & 4.33 & 410.81 & 2.83 \\
\hline 39 & $m$-cresol & training & 523.51 & 5 & 453.8 & 448.36 & -1.2 & 432.17 & -4.77 \\
\hline 40 & $m$-cresol & training & 573.38 & 10 & 587.5 & 579.85 & -1.3 & 568.24 & -3.28 \\
\hline 41 & $m$-cresol & training & 574.48 & 10.2 & 587.2 & 586.69 & -0.09 & 573.94 & -2.26 \\
\hline 42 & $m$-cresol & validation & 598.22 & 13.3 & 830.2 & 817.25 & -1.56 & 784.74 & -5.48 \\
\hline 43 & $m$-cresol & training & 524.38 & 10.1 & 431.9 & 420.37 & -2.67 & 422.76 & -2.12 \\
\hline 44 & $m$-cresol & training & 573.3 & 20.2 & 463 & 451.64 & -2.45 & 465.86 & 0.62 \\
\hline 45 & $m$-cresol & prediction & 598.17 & 20.3 & 612.4 & 586.86 & -4.17 & 608.29 & -0.67 \\
\hline 46 & $m$-стеsol & training & 474.89 & 30.4 & 373.7 & 384.75 & 2.96 & 387.24 & 3.62 \\
\hline 47 & $m$-cresol & training & 523.6 & 30.3 & 387.2 & 396.01 & 2.28 & 397.79 & 2.74 \\
\hline 48 & $m$-cresol & validation & 573.23 & 30.1 & 430.5 & 440.45 & 2.31 & 425,09 & -1.26 \\
\hline 49 & $m$-cresol & training & 573.75 & 29.8 & 433.5 & 441.82 & 1.92 & 426.58 & -1.60 \\
\hline 50 & $m$-cresol & prediction & 598.2 & 30.2 & 490.7 & 508.79 & 3.69 & 480.45 & -2.09 \\
\hline 51 & $m$-cresol & training & 623.17 & 30.2 & 647.9 & 648.69 & 0.12 & 669.99 & 3.41 \\
\hline 52 & $p$-cresol & training & 303.55 & 0.1 & 400.6 & 398.57 & -0.51 & 395.41 & -1.30 \\
\hline 53 & $p$-cresol & validation & 372.24 & 2.2 & 370.4 & 395.42 & 6.75 & 402.43 & 8.65 \\
\hline 54 & $p$-cresol & training & 422.63 & 2.1 & 376.8 & 394.9 & 4.8 & 407.05 & 8.03 \\
\hline 55 & $p$-cresol & training & 473.53 & 2.1 & 397.6 & 412.21 & 3.67 & 412.81 & 3.83 \\
\hline 56 & $p$-cresol & prediction & 523.51 & 5 & 450.7 & 444.8 & -1.31 & 435.46 & -3.38 \\
\hline 57 & $p$-cresol & training & 573.38 & 10 & 579.9 & 596.26 & 2.82 & 583.58 & 0.63 \\
\hline 58 & $p$-cresol & training & 574.1 & 10.1 & 576.3 & 601.48 & 4.37 & 588.13 & 2.05 \\
\hline 59 & $p$-cresol & training & 598.22 & 13.3 & 809.2 & 844.82 & 4.4 & 811.10 & 0.24 \\
\hline
\end{tabular}


Table 1. Continued

\begin{tabular}{|c|c|c|c|c|c|c|c|c|c|}
\hline No. & Aqueous solutions & Data set & $\mathrm{T}$ & $\mathrm{P}$ & $\mathrm{C}_{p .2}^{\circ}(\operatorname{esp})$ & $C_{p, 2}^{\infty}(\mathrm{call})^{\prime \prime}$ & $\mathrm{IPD}^{\prime \prime}$ & $C_{p, 2}^{o}(\text { call })^{b}$ & $\mathrm{IPD}^{b}$ \\
\hline 60 & $p$-cresol & training & 524.38 & 10.1 & 432.1 & 432.14 & 0.01 & 425.78 & -1.46 \\
\hline 61 & $p$-cresol & training & 573.3 & 20.2 & 459.8 & 469.55 & 2.12 & 473.14 & 2.90 \\
\hline 62 & $p$-cresol & prediction & 598.18 & 20.4 & 595.6 & 604.82 & 1.55 & 625.71 & 5.05 \\
\hline 63 & $p$-cresol & training & 474.89 & 30.4 & 367.2 & 385.33 & 4.94 & 393.29 & 7.11 \\
\hline 64 & $p$-cresol & training & 523.6 & 30.3 & 383.6 & 396.88 & 3.46 & 402.51 & 4.93 \\
\hline 65 & $p$-cresol & validation & 573.23 & 30.3 & 423 & $\$ 42.46$ & 4.6 & +29.82 & 1.61 \\
\hline 66 & $p$-cresol & training & 573.78 & 29.9 & 420.7 & 444.19 & 5.58 & 431.68 & 2.61 \\
\hline 67 & $p$-cresol & training & 598.2 & 30.4 & 488 & 512.12 & 4.94 & 488.55 & 0.11 \\
\hline 68 & $p$-cresol & prediction & 623.17 & 29.9 & 622.8 & 658.76 & 5.77 & 702.39 & 12.78 \\
\hline 69 & aniline & training & 303.55 & 0.1 & 336.6 & 342.3 & 1.69 & 321.41 & -4.51 \\
\hline 70 & aniline & validation & 372.24 & 2.2 & 327.5 & 331.28 & 1.15 & 329.98 & 0.76 \\
\hline 71 & aniline & training & 422.61 & 2.1 & 332.4 & 335.59 & 0.96 & 340.48 & 2.43 \\
\hline 72 & aniline & training & 473.53 & 2.1 & 346.8 & 341.84 & -1.43 & 354.56 & 2.24 \\
\hline 73 & aniline & prediction & 523.52 & 5 & 392.2 & 370.13 & -5.63 & 376.53 & -3.99 \\
\hline 74 & aniline & validation & 574.26 & 10.1 & 519.9 & 485.41 & -6.63 & 484.48 & -6.81 \\
\hline 75 & aniline & training & 523.6 & 30.2 & 348.6 & 335.3 & -3.81 & 327.79 & -5.97 \\
\hline 76 & antiline & validation & 573.58 & 29.9 & 384.9 & 352.4 & -8.44 & 350.81 & -8.86 \\
\hline 77 & $o$-toluidne & training & 303.55 & 0.1 & 410.5 & 411.29 & 0.19 & 399.79 & -2.61 \\
\hline 78 & $o$-toluidne & prediction & 372.23 & 2.2 & 405.1 & 407.32 & 0.55 & 404.27 & -0.20 \\
\hline 79 & $o$-toluidne & training & 422.61 & 2.1 & 402.6 & 40003 & -0.64 & 409.15 & 1.63 \\
\hline 80 & $o$-toluidne & training & 473.53 & 2.1 & 431.4 & 406.3 & -5.82 & 415.43 & -3.70 \\
\hline 81 & $o$-toluidne & validation & 523.53 & 5 & 482.1 & 441.88 & -8.34 & 477,07 & -7.27 \\
\hline 82 & $o$-toluidne & training & 573.69 & 10.1 & 638.6 & 640.67 & 0.32 & 663.27 & 3.86 \\
\hline 83 & $o$-toluidne & training & 523.59 & 30 & 418.8 & 399.47 & -4.62 & 407.31 & -2.74 \\
\hline 84 & $o$-toluidne & prediction & 573.73 & 29.8 & 472.7 & 451.19 & -4.55 & 445.92 & -5.66 \\
\hline 85 & $m$-toluidne & training & 303.55 & 0.1 & 406.2 & 405.8 & -0.1 & 396.77 & -2.32 \\
\hline 86 & m-toluidne & validation & 372.2 & 2.1 & 406.1 & 401.42 & -1.15 & 403.40 & -0.67 \\
\hline 87 & $m$-toluidne & training & 422.61 & 2.1 & 393.4 & 396.64 & 0.82 & 407.58 & 3.60 \\
\hline 88 & $m$-toluidne & training & 473.53 & 2.1 & 412.9 & 407.33 & -1.35 & 412.28 & -0.15 \\
\hline 89 & $m$-toluidne & prediction & 523.53 & 5 & 472.3 & 441.54 & -6.51 & 428.38 & -9.30 \\
\hline 90 & $m$-toluidne & validation & 574.22 & 10.1 & 629.5 & 626.98 & -0.4 & 532.74 & -15.37 \\
\hline 91 & $m$-toluidne & training & 523.59 & 30 & 414 & 398.17 & -3.82 & 403.11 & -2.63 \\
\hline 92 & $m$-toluidne & validation & 573.73 & 29.8 & 456.4 & 448.2 & -1.8 & 424.61 & -6.97 \\
\hline 93 & $p$-toluidne & training & 303.55 & 0.1 & 400.2 & 39807 & -0.53 & 392.29 & -1.98 \\
\hline 94 & $p$-toluidne & prediction & 372.24 & 2.2 & 398.7 & 395.03 & -0.92 & 400.27 & 0.39 \\
\hline 95 & $p$-toluidne & training & 422.61 & 2.1 & 395.4 & 394.91 & -0.12 & 405.61 & 2.58 \\
\hline 96 & $p$-toluidne & training & 473.53 & 2.1 & 426.2 & 412.7 & -3.17 & 412.23 & -3.28 \\
\hline 97 & $p$-toluidne & validation & 523.53 & 5 & 476.5 & 445.21 & -6.57 & 437.80 & -8.12 \\
\hline 98 & $p$-toluidne & training & 573.71 & 10.1 & 639.6 & 597.01 & -6.66 & 607.16 & -5.07 \\
\hline 99 & $p$-toluidne & training & 523.59 & 30 & 412.5 & 396.55 & -3.87 & 400.60 & -2.88 \\
\hline 100 & $p$-toluidne & prediction & 573.75 & 29.9 & 462.3 & 443.84 & -3.99 & 433.11 & -6.31 \\
\hline 101 & $m$-anumophenol & training & 304.47 & 0.1 & 285 & 285.34 & 0.12 & 307.12 & 7.76 \\
\hline 102 & m-aniniophenol & validation & 372.23 & 2.2 & 303.2 & 308.51 & 1.75 & 30808 & 1.61 \\
\hline 103 & m-anuinophenol & training & 422.61 & 2.1 & 328.7 & 328.88 & 0.05 & 309.60 & -5.81 \\
\hline 104 & $m$-anuinophenol & training & 473.53 & 2.1 & 332 & 331.87 & -0.04 & 312.64 & -5.83 \\
\hline 105 & m-ancinophentol & prediction & 523.53 & 5 & 342.6 & 295.57 & -13.73 & 320.64 & -6.41 \\
\hline 106 & m-aniniophenol & training & 574.35 & 10.1 & 340.5 & 340.54 & 0.01 & 365.57 & 7.36 \\
\hline 107 & m-aminophenol & prediction & 523.6 & 30.2 & 325.4 & 286.02 & -12.1 & 308.36 & -5.24 \\
\hline 108 & m-aminophenol & validation & 573.55 & 29.9 & 323.8 & 307.03 & -5.18 & 316.05 & -2.39 \\
\hline 109 & $o$-diaminobenzene & training & 303.55 & 0.1 & 386.5 & 386.27 & -0.06 & 388.51 & 0.52 \\
\hline 110 & $o$-diaminobenzene & prediction & 372.25 & 2.2 & 395.8 & 390.46 & -1.35 & 397.57 & 0.45 \\
\hline 111 & $o$-diaminobenzene & training & 422.65 & 2.1 & 417.9 & 406.06 & -2.83 & 403.67 & -3.41 \\
\hline 112 & $o$-diaminobenzene & training & 473.55 & 2.1 & 433.9 & 437.4 & 0.81 & 410.13 & -5.48 \\
\hline $11 \hat{3}$ & o-dianninobenzente & validation & 523.55 & 5 & 465 & 461.28 & -0.8 & 430.10 & -7.50 \\
\hline 114 & $o$-diaminobenzente & prediction & 574.55 & 10.2 & 525.3 & 517.23 & -1.54 & 560.65 & 6.73 \\
\hline 115 & $o$-diaminobenzente & training & 523.65 & 30.2 & 426.1 & 392.31 & -7.93 & 396.62 & -6.92 \\
\hline 116 & $o$-diaminobenzene & prediction & 573.75 & 30 & +43.2 & 431.98 & -2.53 & 423.31 & -4.49 \\
\hline
\end{tabular}

"The calculated values of $\mathrm{C}_{p, 2}^{0}$ and IPD using the ANN model with architecture 3-9-1. ${ }^{3}$ The calculated falues of $\mathrm{C}_{p, 2}^{0}$ and IPD using the ANN model with architecture 6-3-1. 


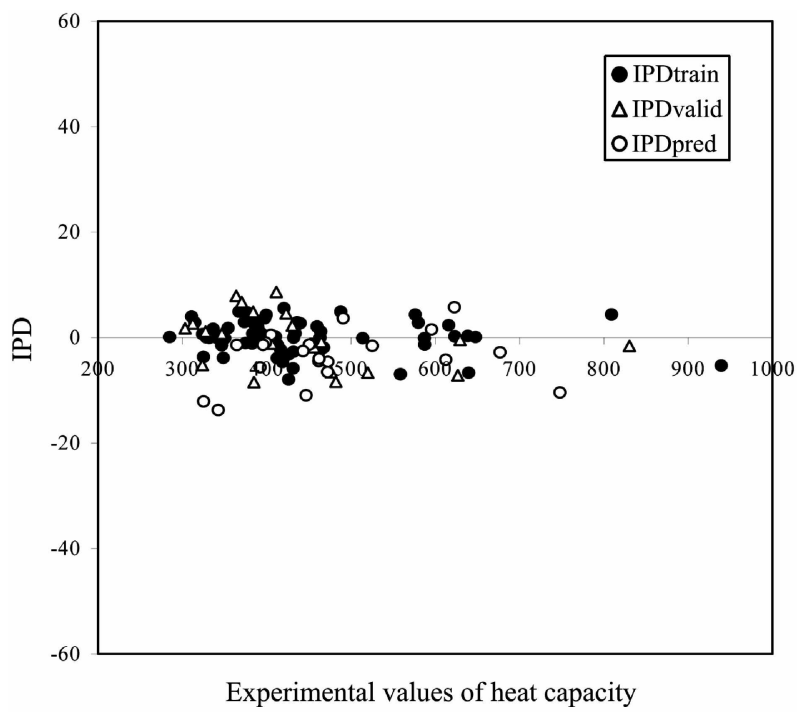

Figure 3. Plot of the IPI) (individual percent deviation) for calculated values of the heat capacity from the $A N N$ model wilh architecture 3-6-1 versus the experimental values of it for training, validation and prediction sets.

Table 2. Statistical parameters obtained by the $A N N$ model with architecture 3-9-1 for total, training. validation and prediction sets ${ }^{a}$

\begin{tabular}{lrccrr}
\hline Type of data set & $\mathrm{N}$ & $\mathrm{R}$ & $\mathrm{MPI}$ & $\mathrm{RMSL}$ & \multicolumn{1}{c}{$\mathrm{F}$} \\
\hline Total & 116 & 0.9859 & 3.017 & 19.642 & 3950.35 \\
Training & 74 & 0.9915 & 2.163 & 14.608 & 4155.31 \\
Vadidation & 21 & 0.9841 & 4.262 & 22.975 & 584.37 \\
Prediction & 21 & 0.9761 & 4.755 & 29.179 & 383.29 \\
\hline
\end{tabular}

" $\mathrm{Y}$ is number of datal set: $\mathrm{R}$ is the correlation coefficient between calculated and the experimental values of the partial molar heat capicity at inlinite dilution: MPL) is mean percent deviation: RMSE is root mean square error and $f$ is the statistical li-valuc.

control the overtraining of the network during the training procedure, the values of RMSET and RMSEV were calculated and recorded to monitor the extent of the learning in various iterations. Results obtained show that overfitting does not exist for this ANN and training is stop after 80000 iterations (Figure 5).

For evaluation predictive power of the generated ANN, an optimized network was applied for prediction the heat capacity of different aqueous solutions at various temperatures and pressures in the prediction set.

Values of the partial molar heat capacity for different aqueous solutions of various polar aromatic compounds along with the calculated and IPD values at various temper-

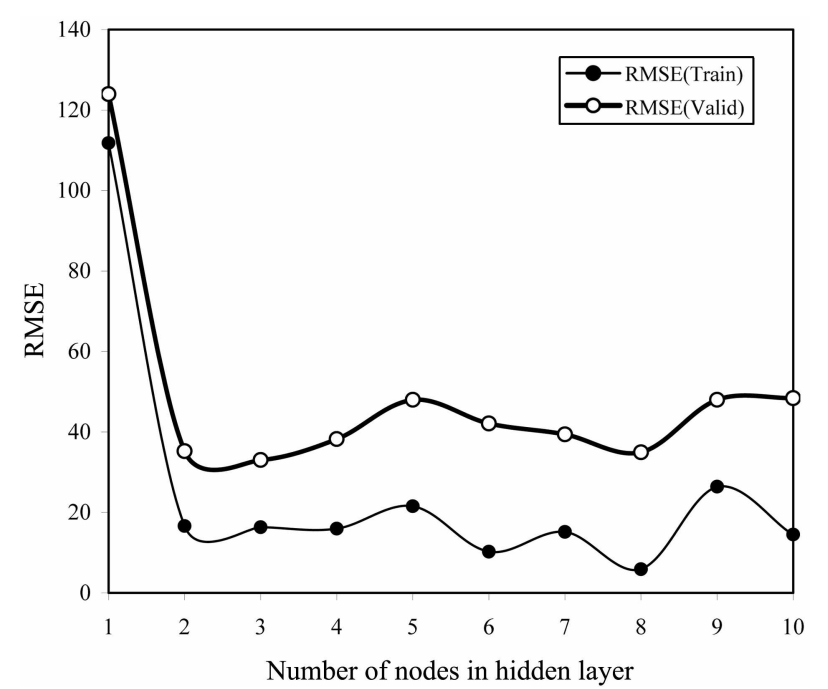

Figure 4. Plot of RMSF, for training and validation sets wersus the number of nodes in hidden layer.

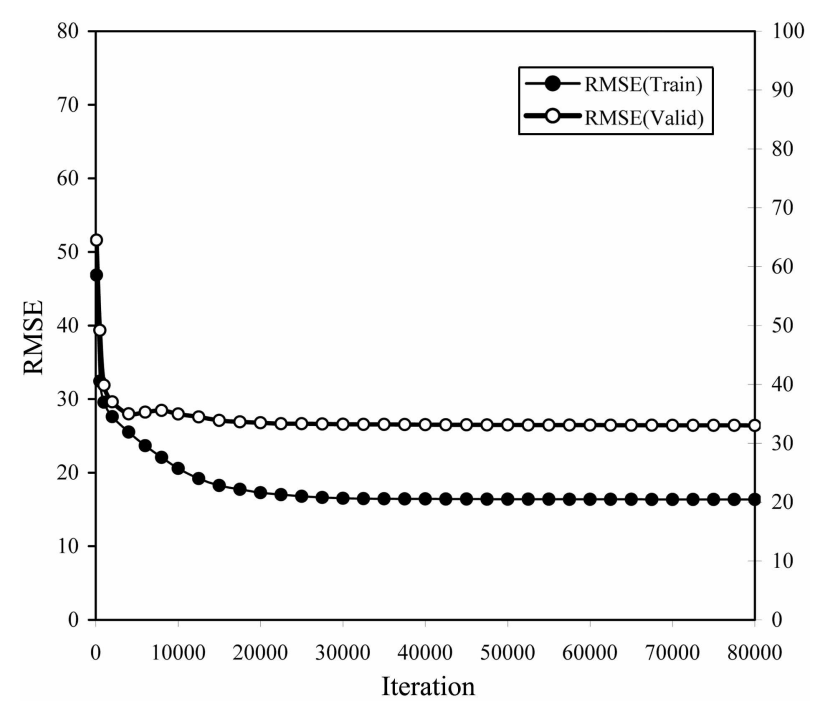

Figure 5. Plot of RMSF, for training and validation sets (for the A.N model with architecture 6-3-1) versus the number of itcrations.

atures and pressures for training, validation and prediction sets have been shown in Table $\mathrm{l}$.

As can be seen the calculated values of the heat capacity are in good agreement with those of the experimental values. The correlation equation for all of the calculated values of the heat capacity from the ANN model and the experimental

Table 3. Theoretical descriptors, symbols and coefficients in the VI. R model

\begin{tabular}{|c|c|c|}
\hline Name of descriptor & Symbol & Coefficient \\
\hline Complementary information content (ncighborhood symmetry of 0 -order) & $\mathrm{CICO}$ & 382.718 \\
\hline Geary autocorrelation-lag3/ucighted by atomic masses & GATS3m & -16751.82 \\
\hline Radial distribution function- 5.0 weighted by atomic masses & RDF050m & -12.754 \\
\hline 3D-MoRSF-signal 08/ueighted by atomic polarizabilities & Mor08p & -181.68 \\
\hline Constant & & -433.178 \\
\hline
\end{tabular}




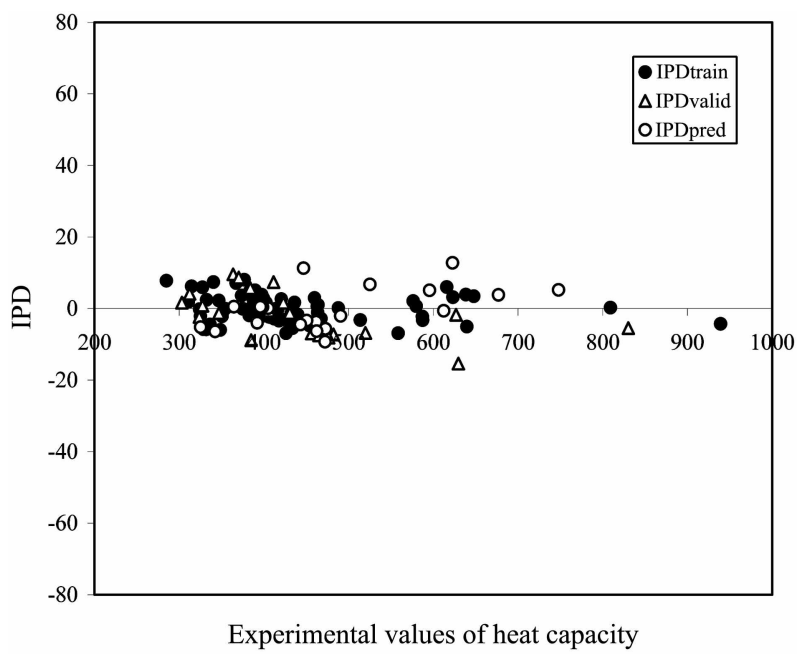

Figure 6. Plot of the [PJ) (individual percent deviation) for calculated values of the heal capacity from the $A N N$ model with architecture 6-3-I versits the experimental values of it for training. validation and prediction sets.

Table 4. Statistical parameters obtained by the $\Lambda N N$ model with architecture 6-3-1 for total, training. validation and prediction sets ${ }^{a}$

\begin{tabular}{lrcccc}
\hline Type of datal set & $\mathrm{N}$ & $\mathrm{R}$ & $\mathrm{MPI}$ & $\mathrm{RMSl}$ & $\mathrm{F}$ \\
\hline Total & $\mathrm{I} 16$ & 0.9800 & 3.819 & 23.015 & $2762.7 \mathrm{I}$ \\
Traiting & 74 & 0.9893 & 3.141 & 16.348 & 3305.60 \\
Validation & 21 & 0.9745 & 5.386 & 33.028 & $358.6 \mathrm{I}$ \\
Prediction & $2 \mathrm{I}$ & 0.9815 & 4.642 & 29.885 & 498.20 \\
\hline
\end{tabular}

$\mathrm{A}$ is number of datia set: $\mathrm{R}$ is the correlation coefficient between calculated and the experimental values of the partial molar heat capicity at inlinite dilution: MPL) is mean percent deviation: RMSE is root mean square error and $t$ is the statistical li-valuc.

values is as follows:

$$
\begin{gathered}
\mathrm{C}_{r, 2}^{\prime \prime}(\mathrm{cal})-0.9760 \mathrm{C}_{f, 2}^{\prime \prime}(\mathrm{exp})+8.894 \\
\mathrm{~N}=116 ; \mathrm{R}=0.9800 ; \mathrm{MPD}=3.819 \\
\mathrm{RMSE}=23.015 ; \mathrm{H}=2762.71
\end{gathered}
$$

Similarly, the correlation of $\mathrm{C}_{p, 2}^{\prime \prime}$ (cal) values verste $\mathrm{C}_{p, 2}^{\prime \prime}$ (exp) in prediction set gives equation (8):

$$
\begin{gathered}
\mathrm{C}_{p, 2}^{\prime \prime}(\mathrm{cal})=1.154 \mathrm{C}_{7,2}^{\prime \prime}(\exp )-71.783 \\
\mathrm{~N}-21 ; \mathrm{R}-0.9815: \mathrm{MPD}-4.642 \\
\text { RMSE - 29.885: F }-498.20
\end{gathered}
$$

The results demonstrate that the $\mathrm{MPD}$ value for $\mathrm{C}_{p, 2}^{\prime \prime}$ values in the prediction set is 4.642 .

Plot of IPD for Cp values in prediction set versus the experimental values of it has been illustrated in rigure 6. As can be seen the model did not show proportional and systematic error, because the propagation of errors in both sides of zero are random.

The correlation coefficient (R), RMSE, MPD and statistical F-value of the model for total, training, validation and prediction sets show potential of the ANN model for prediction the heat capacity of the aqueous solutions at various temperatures and pressures ( lable 4).
As a result. it was found that the properly selected and trained neural networks could fairly represent the dependence of the heat capacity of the aqueous solutions on theoretical descriptors, temperatures and pressure.

\section{Conclusions}

Two types of inputs have been applied for prediction partial molar heat capacity of aqueous solutions at infinite dilution for various polar aromatic compounds (including phenol, $o$-cresol, $m$-cresol, $p$-cresol, aniline, $o$-toluidine, $m$ toluidine, $p$-toluidine, $m$-aminophenol, $p$-aminophenol and $o$-diaminobenzene) over wide range of temperatures ( 303.55 $-623.20 \mathrm{~K})$ and pressures (0.1-30.2 MPa) using artificial neural network models. In these models macroscopic and microscopic properties of the compounds along with temperature and pressure have been used as inputs and their output is the partial molar heat capacity. The MPD values of the models for prediction set are 4.755 and 4.642, respectively. Then the optimized neural network could simulate the complicated nonlinear relationship between the partial molar heat capacity for various polar aromatic compounds on the heat capacity in $\mathrm{I}=303.55 \mathrm{~K}$ and $\mathrm{P}=0 . \mathrm{I} \mathrm{MPa}$ (or theoretical inolecular descriptors), temperature and pressure. As a result ANNs can be used to predict the heat capacity at higher temperatures and pressures using minimum number of experiments.

Acknowledgements. The Authors wish to acknowledge the vice presidency of research, university of Mohaghegh Ardebili, for financial support of this work.

\section{References}

1. Censky. M.: Hnedkowshy. L.: Majer. V. I. Chen. Thermodyn. 2004. 37. 203.

2. Censky. M.: Intedkovsky. 1.: Majer. V. /. Chem. Thennodn. $2005,37.225$

3. Katritzky. A. R.: Karelson. M.: lobanov. V. S. Pare ippl. (hem. 1997. 69. 245 .

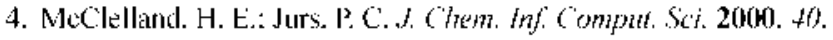
967.

5. Ilabibi-Yangich. A. Indian J. Chem. B 2004, $\$ 3,1504$.

6. Cronce, D. T.: Famini, (i R: Soto, J. A. D.: Wilson, I., Y, $J$. (h'm. Soc Perkin Trans. 2 1998. 1293.

7. Engherts. I. B. F. N.: Famini. G. R.: Perjessy. A.: Wilson. L. Y. J. Phis. Org. Chem. 1998. /1.26].

8. Nikolic. S.: Milicevic. A.: Trinajstic. N.: Juric. A. Wolecutes 2004. 9. 1208.

9. Devillers, J. SiR and OSAR EMiron. Res. 2004, 15.501.

10. Karelson. M.: Lobanov. V. S. (hem. Rev: 1996. 96. 1027.

11. Jodeschini. R.: Consonni. V. Hardibook of Wolecular Descriptors: Wiley-VCH: Weinheim. 2000.

12. Kratnor. R. Chenontetric Techniques for Otantitative Anthisis: Marcel Dekiker: New York, 1998.

13. Kulzmanovski, I.: Aleksovska S. Chemom. Intelf. Lab. Syst 2003. 67.167.

14. Barros. A. S.: Rutledge. D. ‥ Chemonet Intell Lab. Syst 1998. 40. 65 .

15. Garkani-Nejad. Z:: Karlovits. M.: Demuth. W.: Stimptl. T:: Vycudilik, W.: Jalali-H leravi, M.: Varmuzis K. .J. Chromatogr A 
2004. 1028.287.

16. Bose. N. K.: Liang. P. Neural Network Futdantentals: McGrawHill: New York. 1996.

17. Zupan. I.: Gasteiger J. Neural Networks in Chemisty and Drug Design, Wiley-VCH: Weinhein, 1999.

18. Agatonotic-Kustrin. S.: Beresford, R. J. Pham Bioned And. 2000. 22.717.

19. Despagne. F.: Massart. D. L. Anatyst 1998. 123. 157R.

20. Borosy. A. P.: Balogh. B.: Matyus. P. J. hol. Srtuc.(Theochemi 2005, 729, 169.

21. Bunz, A. P.: Braun. B.: Janowsky, R. F/uid Phase Equilib. 1999. 158,367

22. Homer. J.: Generalis, S. C.: Robson. J. H. Phws. Chem. Chem. Plws. 1999. I. 4075.

23. Goll. E. S.: Jurs. P. C. J. Chem Inf. Contp. Sci. 1999.39.974

24. Vendrame R.: Braga. R. S: Takahata, Y.: Galvao. D. S. J. Chem. Inf. Comput. Sci. 1999, 39. 1094.

25. Gaspelin. M.: Tusar. L.: Smid-Korbar. J.: Zupan. J.: Kristl. J. Int. J. Pharm. 2000. 196.37.

26. Gini. G.: Cracium. M. V.: Konig. C.: Bentenati. E. J. Chem. Inf. Comput Sci. 2004. H. 1897.

27. Urata, S: Takada A: Uchimaru, T.: Chandra. A. K.: Sekiva. A.J. Fhorine Chem. 2002, 116. 163.

28. Koziol. J. Intemet Electron. J. M Iol Des. 2002, 1,80.

29. Wegner. J. K: Zell. A. J. Chem. Inf. Comput Sit. 2003. 13. 1077.

30. Valkova. I: Vracko. M.: Basak. S. C. Anat Chim. Acta 2004. 509. 179.

31. Sebastiao. R. C. O) Braga, I. P.: Yoshida, M. I. Thermodhinica Acta 2004. 112, 107 .
32. Jalali-Heravi. M.: Masoum. S.: Shahbazikhah. P. J. Magn. Rexon. 2004. 17]. 176.

33. Habibi-Yangjeh. A.: Nooshyar. M. Bull. Korean Chent. Soc. 2005. 26. 139 .

34. Habibi-Yangeh, A: Nooshyar. M. Physics and Chemistry of Liquids 2005. 13. 239.

35. Habibi-Yangieh. A.: Danandeh-Jenagharad. M.: Nooshyar. M. Bull. Korean Chen. Soc. 2005.26 .2007$.

36. Habibi-Yangjeh. A.: Danandeh-Tenagharad. M.: Nooshyar. M. J. Ifol. Model. 2006, 12.338.

37. Habibi-Yangjeh, A.: Danandeh-Jenagharad, M. Indian J. Chem. B 2007. 46,478

38. Habibi-Yangjeh. A. Plnsics and Chemistry of Liquids (in press).

39. Guha. R.: Turs. P. C. J. Chem. Inf. Comput Sei. 2004. H. 1440.

40. Mosier. P. D.: Countermant. A. E.: Turs. P. C.: Clemmer. D. E. Anal. Chem. 2002, 74. 1360.

41. Guha. R.: Jurs. P. C. J. Chem. Inf M lodel 2005, 45.800.

42. HyperChem. Release 7.0 for Windows. Holecular Modeling Sustent. Hypercube Inc: 2002.

43. Todeschini. R.: Consonni. V.: Pavan. M. Dragon Sofnore Iersion 2.1:2002.

H. Hemmateenejad, B; Alhond. M.: Miri, R:; Shamsipur. M. $d$. Chem. Inf. Comput. Sci. $2003,43,1328$

45. Hemmateenejad, M: Safarpour. M. A.: Miri, R.: Nesari, N. J. Chent. Inf. Hodel. 2005. 45.190

46. SPSS for windon's. Release 100: SPSS Inc.: 1989-1999.

47. Demuth. H.: Beale. M. Neural Network Toolbox: Mathworks: Natick, MA, 2000.

48. Matlab 6.5. Mathworks 1984-2002. 